Communications in Physics, Vol. 19, No. 4 (2009), pp. 193-200

\title{
EFFECT OF SPONTANEOUS POLARIZATION CHARGES ON THE ELECTRON MOBILITY IN ZnO SURFACE QUANTUM WELLS
}

\author{
NGUYEN THANH TIEN \\ College of Science, Cantho University \\ LE TUAN \\ Institute of Engineering Physics, Hanoi University of Technology \\ DOAN NHAT QUANG \\ Institute of Physics, VAST
}

\begin{abstract}
We present a theoretical study of the effect due to spontaneous polarization of $Z n O$ on the low-temperature mobility of the two-dimensional electron gas (2DEG) in a ZnO surface quantum well (SFQW). We proved that for the O-polar face this causes an attraction of electrons by the positive charges bound on the surface, while for the Zn-polar face a repulsion of them far away therefrom by the negative bound charges of the same magnitude. Accordingly, surface roughness scattering is drastically enhanced in the former case, but reduced in the latter one. Therefore, the low-temperature 2DEG mobility in ZnO SFQWs with O-polar face is found to be dominated by surface roughness. Our theory was illustrated for the sample prepared by bombardment of the O-polar face by 100-eV hydrogen ions. The surface roughness scattering enables an explanation of the 2DEG mobility, especially, the reason of low values for the mobility in the dependence from the carrier density which has not been understood when starting from impurity scattering.
\end{abstract}

\section{INTRODUCTION}

The semiconductor $\mathrm{ZnO}$ has gained substantial interest in the research community in part because of its large exciton binding energy $(60 \mathrm{meV})$ [1-4] which could lead to lasing action based on exciton recombination even above room temperature. Even though research focusing on $\mathrm{ZnO}$ goes back many decades, the renewed interest is fueled by availability of high-quality substrates and reports of p-type conduction and ferromagnetic behavior when doped with transitional metals, both of which remain controversial.

It was experimentally indicated that 2DEG is formed at the naked surface of semiconductor $\mathrm{ZnO}$ [5-8]. This open structure is referred to as a surface quantum well, in which a very high potential barrier $(\sim 5 \mathrm{eV})$ between the vacuum and the host crystal leads to an enhanced electron confinement. For ion-implanted $\mathrm{ZnO}$, the theoretical $[9,10]$ and experimental [11] studies stipulate a Gaussian distribution for the impurities. It was pointed out [12-14] that $\mathrm{ZnO}$ in the natural (wurtzite) phase with low symmetry has a large spontaneous polarization $\left(P_{s p}=-0.057 \mathrm{Cm}^{-1}\right.$, twice of that of $\mathrm{GaN}$, it is equivalent to a negative sheet carrier density on of $\sigma_{p} / e=-3.6 \times 10^{13} \mathrm{~cm}^{-2}$ at the $\mathrm{Zn}$-polar face and a bound positive sheet carrier density of the same magnitude at the O-polar face) along the c direction. This must exert some influence on the electron transport in ZnO-based 
structures, however, scarcely studied. Recently, we have proved [15] that ionic correlation reduces remarkable impurity scattering, so that for annealed ZnO SFQWs ionized donors might be not a key scattering mechanism. On the other hand, we have shown $[16,17]$ that polarization charges bound on an interface of GaN-based heterostructures may bring about a remarkable shift of the electronic distribution toward this interface, so an enhancement of surface roughness scattering. Therefore, surface roughness scattering strengthen by spontaneous polarization in $\mathrm{ZnO}$ SFQWs is expected to be violent.

Thus, the aim of this paper is to present a theory of the effect of spontaneous polarization on the 2DEGs mobility in a $\mathrm{ZnO}$ SFQW. We will treat the low-temperature mobilites of electrons confined in a Gaussian heavily doped $\mathrm{ZnO}$ surface quantum wells. Especially, it will be shown an illustration of the relation between the distribution of the 2DEGs and its partial mobilities.

\section{TWO-DIMIENSIONAL ELECTRON GAS IN A GAUSSIAN-DOPED SFQW WITH SPONTANEOUS POLARIZATION}

\section{II.1. Confining potentials}

We examine the effect from a Gaussian doping, especially by the spontaneous polarization on the distribution of electrons in a $\mathrm{ZnO}$ SFQWs. The quantum confinement along the $z$ direction (normal to the surface) is determined by the following Hamiltonian:

$$
H=T+V_{\text {tot }}(z)
$$

where $T$ is the kinetic energy, and $V_{\text {tot }}(z)$ is the effective confining potential given by

$$
V_{\mathrm{tot}}(z)=V_{b}(z)+V_{H}(z)+V_{p}(z)+V_{\mathrm{im}}(z)+V_{\mathrm{xc}}(z) .
$$

Here, the first term is due to a potential barrier located at the surface plane $z=0$. The second term is Hartree potential due to ionized donors and confined electrons themselves. The third term in Eq. (2) is the potential due to spontaneous polarization charges bound on the $\mathrm{ZnO}$ surface $(z=0)$, so that

$$
V_{p}(z)=\frac{2 \pi e^{2}\left(\sigma_{p} / e\right)}{\varepsilon_{L}} z,
$$

with $\sigma_{p} / e$ as their sheet density. The fourth term is a classical repulsion potential due to image charge, which quantifies the effect arising from an abrupt decrease in the dielectric constant across the surface $z=0$. At last, the exchange-correlation corrections allow for the many-body effect in the 2DEG along the normal direction.

In what follows, we are concerned with charges and their electric fields in the $\mathrm{ZnO}$ side. The 2DEG in the lowest subband of a ZnO SFQW is clearly described by a standard Fang-Howard wave function [18]:

$$
\zeta(z)=\left(k^{3} / 2\right)^{1 / 2} z e^{-k z / 2}
$$

in $\mathrm{ZnO}(z \geq 0)$ and equal to zero in the vacuum $(z<0)$. Here, $k$ is the wave number to be determined. 
It was pointed out [9-11] that the donor density distribution in $\mathrm{ZnO}$, especially under hydrogen-ion bombardment, is of Gaussian shape with a peak at some point $z_{D}>0$, so that

$$
N_{D}(z)=\frac{n_{D}}{\sigma \sqrt{2 \pi}} \exp \left[-\left(\frac{z-z_{D}}{\sigma \sqrt{2}}\right)^{2}\right],
$$

in $\mathrm{ZnO}$ and equal to zero in the vacuum. Here, $\sigma$ is a standard deviation of the Gaussian function.

The electron density distribution in $\mathrm{ZnO}$ is specified by the wave function from Eq. (4):

$$
n(z)=n_{s}|\zeta(z)|^{2}
$$

where $n_{s}$ is a sheet density of electrons.

\section{II.2. Total energy per electron in the lowest subband}

We now turn to the total energy per particle in the ground-state subband, which is to be minimized to find the wave number $k$ entering in Eq. (4). Within the infinite-barrier model: $\left\langle V_{b}\right\rangle=0$, the expectation value of the Hamiltonian is given by

$$
E(k)=\langle T\rangle+\left\langle V_{D}\right\rangle+\left\langle V_{s}\right\rangle+\left\langle V_{p}\right\rangle+\left\langle V_{\mathrm{im}}\right\rangle+\left\langle V_{\mathrm{xc}}\right\rangle .
$$

The total energy per electron is obtained by a modification of Eq. (7), in which the average 2DEG potential $\left\langle V_{s}\right\rangle$ is to be replaced with its half [18].

Upon making use of the above-derived expressions for the partial confining potentials, we are able to estimate their expectations in the electronic state described by the wave function from Eq. (4). The average energies present in Eq. (7) are supplied in [19]. Espectially, we add the average energie by the polarization charges, it holds

$$
\left\langle V_{p}\right\rangle=\frac{2 \pi e^{2}\left(\sigma_{p} / e\right)}{\varepsilon_{L}} \frac{3}{k} .
$$

\section{LOW-TEMPERATURE ELECTRON MOBILITY}

\section{III.1. Basic equations}

In this section we are dealing with the low-temperature mobility of the electrons confined in a Gaussian heavily-doped ZnO SFQWs. At very low temperature the mobility is determined via the momentum relaxation time $\tau$ by a familiar relation

$$
\mu=e \tau / m^{*}
$$

with $m^{*}$ as the in-plane effective electron mass of $\mathrm{ZnO}$.

As known [20], within the linear transport theory, the inverse transport lifetime is represented in terms of the autocorrelation function $\left\langle|U(q)|^{2}\right\rangle$ for each scattering mechanism by

$$
\frac{1}{\tau}=\frac{1}{2 \pi \hbar E_{F}} \int_{0}^{2 k_{F}} d q \frac{q^{2}}{\left(4 k_{F}^{2}-q^{2}\right)^{1 / 2}} \frac{\left\langle|U(q)|^{2}\right\rangle}{\varepsilon^{2}(q)} .
$$

where $q=2 k_{F} \sin (\theta / 2)$ as the $2 \mathrm{D}$ momentum transfer by a scattering event in the $x-y$ plane, with $\theta$ as a scattering angle. The Fermi energy is given by $E_{F}=\hbar^{2} k_{F}^{2} / 2 m^{*}$, with $k_{F}$ as the Fermi wave number fixed by the 2DEGs density: $k_{F}=\sqrt{2 \pi n_{s}}$. 
The dielectric function $\varepsilon(q)$ entering in Eq. (10) takes account of the screening of a scattering potential by the 2DEGs.

At very low temperature scattering by phonons is negligibly weak, so that the electrons confined in a $\mathrm{ZnO}$ SFQWs are expected to experience the following scattering sources located near the surface: ionized donors (ID) and surface roughness (SR). The overall transport lifetime is determined by the ones for the partial scatterings according to the Matthiessen's rule:

$$
\frac{1}{\tau_{\text {tot }}}=\frac{1}{\tau_{I D}}+\frac{1}{\tau_{S R}}
$$

\section{III.2. Autocorrelation function for surface roughness scattering mechanism}

In accordance with Eqs. (9) and (10), for calculating the 2DEG mobility we must derive the autocorrelation functions for the above-quoted scattering mechanisms. The autocorrelation function for scattering from a distribution of ionized donors is quoted from [15]. The autocorrelation function for surface roughness scattering is fixed by the local value of the wave function at the surface plane [18]. We have

$$
U_{S R}(\mathbf{q})=V_{0}|\zeta(0)|^{2} \Delta_{\mathbf{q}}
$$

where $\Delta_{\mathbf{q}}$ denotes a Fourier transform of the surface roughness profile.

Upon replacing the effective confining potential with Eq. (2), we may represent the local value of the wave function in terms of the expectation values of the electric fields created by the partial confining sources [17]:

$$
V_{0}|\zeta(0)|^{2}=\left\langle V_{D}^{\prime}\right\rangle+\left\langle V_{s}^{\prime}\right\rangle+\left\langle V_{p}^{\prime}\right\rangle+\left\langle V_{\mathrm{im}}^{\prime}\right\rangle+\left\langle V_{\mathrm{xc}}^{\prime}\right\rangle,
$$

with $V^{\prime}=\partial V(z) / \partial z$.

Next, by putting Eq. (13) into Eq. (12), we arrive at the autocorrelation function for surface roughness:

$$
\left\langle\left|U_{S R}(q)\right|^{2}\right\rangle=\left|\left\langle V_{D}^{\prime}\right\rangle+\left\langle V_{s}^{\prime}\right\rangle+\left\langle V_{p}^{\prime}\right\rangle+\left\langle V_{\mathrm{im}}^{\prime}\right\rangle+\left\langle V_{\mathrm{xc}}^{\prime}\right\rangle\right|^{2} \times\left\langle\left|\Delta_{\mathbf{q}}\right|^{2}\right\rangle .
$$

Thus, we must evaluate the average electric fields appearing in Eqs. (14). The calculation is straightforward by means of the wave function from Eq. (4). The result is listed below.

For the Gaussian doping of sheet donor density $n_{D}$ :

$$
\left\langle V_{D}^{\prime}\right\rangle=-\frac{4 \pi e^{2}}{\varepsilon_{L}} \frac{n_{D}}{2}\left\{1-\frac{a^{3}}{2} e^{-\delta a}\left[G_{2}(a, \delta)-2 \delta G_{1}(a, \delta)+\delta^{2} G_{0}(a, \delta)\right]\right\} .
$$

For the 2DEG of sheet density $n_{s}$ :

$$
\left\langle V_{s}^{\prime}\right\rangle=\frac{4 \pi e^{2}}{\varepsilon_{L}} \frac{n_{s}}{2} .
$$

For polarization charges:

$$
\left\langle V_{p}^{\prime}\right\rangle=\frac{2 \pi e^{2}\left(\sigma_{p} / e\right)}{\varepsilon_{L}} .
$$

For the image potential:

$$
\left\langle V_{\mathrm{im}}^{\prime}\right\rangle=-\frac{\varepsilon_{-}}{\varepsilon_{+}} \frac{e^{2}}{\varepsilon_{L}} \frac{k^{2}}{8}
$$


Lastly, for exchange-correlation corrections:

$$
\left\langle V_{\mathrm{xc}}^{\prime}\right\rangle=-0.611 \frac{9}{128}\left[2 \Gamma\left(\frac{8}{3}\right)-\frac{3}{4} \Gamma\left(\frac{11}{3}\right)\right] \frac{e^{2}}{\varepsilon_{L}}\left(\frac{n_{s} k^{4}}{2 \pi}\right)^{1 / 3} .
$$

As seen from Eq. (14), surface roughness scattering is specified by the surface profile. This is normally written as:

$$
\left\langle\left|\Delta_{\mathbf{q}}\right|^{2}\right\rangle=\pi \Delta^{2} \Lambda^{2} F_{S R}(t),
$$

where $\Delta$ is a roughness amplitude, and $\Lambda$ a correlation length. The roughness form factor is given by [21]:

$$
F_{S R}(t)=\frac{1}{\left(1+\lambda^{2} t^{2} / 4 n\right)^{n+1}},
$$

where $n$ is an exponent fixing its falloff at large momentum transfer $t$, and $\lambda=\Lambda / \sigma \sqrt{2}$ a dimensionless correlation length.

\section{RESULTS AND DISCUSSIONS}

In what follows, we will apply the foregoing theory to understand the transport properties of Gaussian-doped SFQWs formed on the surface of $\mathrm{ZnO}$ by bombardment with $\mathrm{H}_{2}^{+}$ions. In particular, we provide a quantitative description of the measured dependence of the surface 2DEGs mobility on the carrier density. For numerical calculation, we need to determine the material parameters as input. For $\mathrm{ZnO}$ the dielectric constant is $\varepsilon_{L}=8.2$. As known, the out of-plane effective electron mass is given as $m_{z}=0.28 m_{e}$, whereas the in-plane one depends on the carrier density owing to nonparabolicity of the conduction band at large energies [15], and its diffusion freezing temperature $T_{0}$ is taken to be of the order of the annealing one $\left(T_{0}=700 \mathrm{~K}\right)$.

We are now dealing with the influence arising from spontaneous polarization on the behavior of the 2DEG in Gaussian-doped ZnO SFQWs. We first examine the effect on quantum confinement. In Fig. 1, we display, following Eqs. (4) and (6), the bulk electron density distribution $n(z)$ along the normal direction in the absence and the presence of spontaneous polarization for the O-polar and Zn-polar faces. The doping is specified with a high density $n_{D}=10^{14} \mathrm{~cm}^{-2}$, a standard deviation $\sigma=12 \AA$, and a peak position $z_{D}=7 \AA[10]$. There, the Gaussian bulk donor density distribution $N_{D}(z)$ is also sketched following Eq. (5). It is clearly seen from Fig. 1 that the electron distribution in $\mathrm{ZnO}$ SFQWs depends drastically on spontaneous polarization. The 2DEG is to be shifted toward the surface and its peak is raised in a sample of O-polar face $(000 \overline{1})$, whereas it shifted far away therefrom and its peak is reduced in a sample of Zn-polar face (0001). As a result, the overlap between the electron and donor distributions is increased in the former case, but decreased in the latter one. It is to be stressed that owing to Coulomb repulsion of the polarization charges, the $\mathrm{ZnO}$ SFQW of Zn-polar face can be formed only at very high doping levels. Evidently, the negative polarization charges on this face tend to push 2DEG far away into the host crystal, and to disperse it. Next, we evaluate the effect on the mobility determined by Eqs. (9) and (10) for the scattering mechanisms of interest for the 2DEG confined in ZnO SFQWs Gaussian-doped with the same doping 


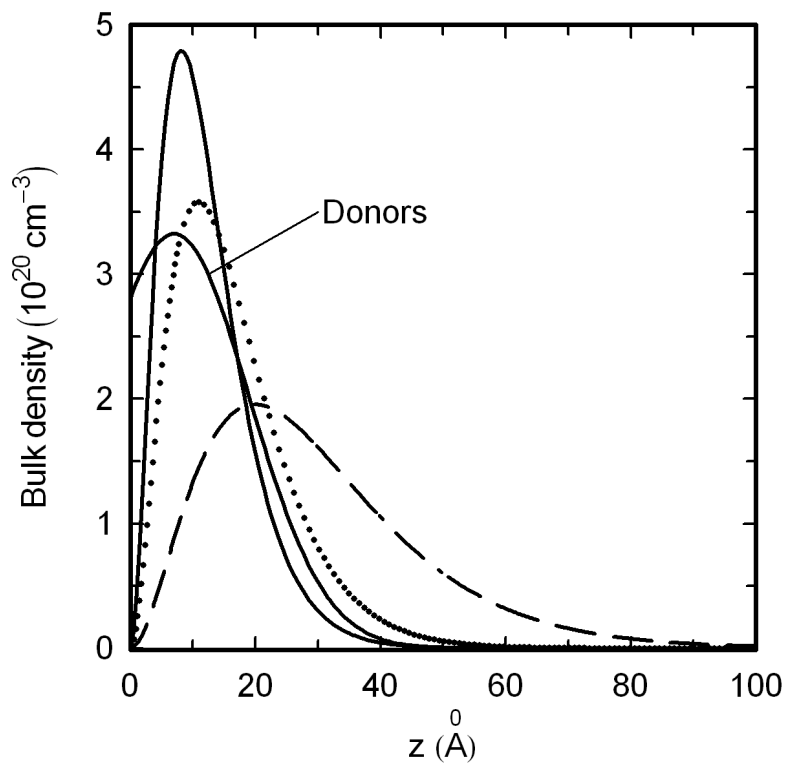

Fig. 1. Bulk density distribution $n(z)$ along the quantization direction for the 2DEG confined in a ZnO SFQW Gaussian-doped with a density $n_{D}=10^{14} \mathrm{~cm}^{-2}$, a standard deviation $\sigma=12 \AA$, and a peak position $z_{D}=7 \AA$. The electron density distribution is displayed without (dotted line) and with spontaneous polarization for the O-polar face (solid line) and Zn-polar face (dashed one). The bulk donor density $N_{D}(z)$ is also shown (solid line).

profile as in Fig. 1. The mobility limited by correlated ionized donors (thermal treatment) $\mu_{I D}$ as a function of electron density $n_{s}$ is plotted in Fig. 2(a). In Fig. 2(b), the mobility limited by surface roughness $\mu_{S R}$ for the ZnO SFQWs. An inspection of Fig. 2(a) reveals that spontaneous polarization gives rise to a decrease of the donor-limited mobility in the O-polar face sample but an increase in the Zn-polar one. This is connected with, as mentioned above, an increase in overlapping between electron and donor distributions in the former case and a decrease in the latter one. The effect is somewhat larger than that in a modulation-doped sample, where the electrons and donors are separated in space, so the polarization effect on impurity scattering is weaker [16].

Figure 2(b) indicates a similar effect of spontaneous polarization on the mobility limited by surface roughness. This is connected with the shift of the electron distribution toward the surface for the O-polar face sample and in the opposite direction for the Znpolar face one. This means that the accumulation layer of O-polar face is of lower quality than that of Zn-polar one. As an example, the surface roughness mobility in an O-polar face sample is decreased by factor of 8 compared to the corresponding $\mathrm{Zn}$-polar face one at $n_{s} \sim 12 \times 10^{13} \mathrm{~cm}^{-2}$.

It is observed from Figs. 2(a) and 2(b) that the polarization effect on both scattering mechanisms is decreased with a rise of the electron density. In addition, the effect on 

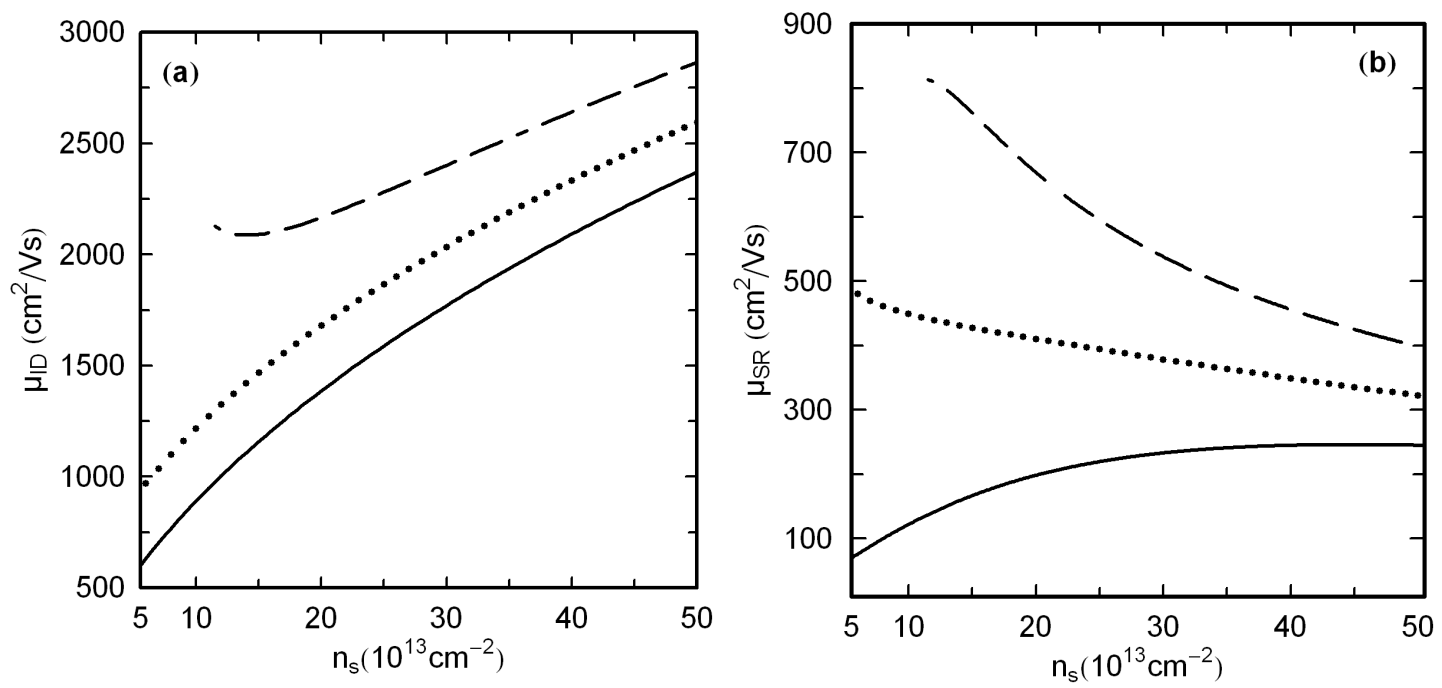

Fig. 2. Mobilities limited by ionized donors $\mu_{I D}$ (Fig a)and by surface roughness $\mu_{S R}$ (Fig b) of the 2DEG in a ZnO SFQW Gaussian-doped with the same doping profile as in Fig. $1\left(\sigma=12 \AA\right.$ and $\left.z_{D}=7 \AA\right)$ vs sheet electron density $n_{s}$ in the absence (dotted lines) and the presence of spontaneous polarization for the O-polar face (solid lines) and Zn-polar face (dashed ones). The surface profile is with an exponent $n=1$, a roughness amplitude $\Delta=10 \AA$, and correlation length $\Lambda=30 \AA$.

surface roughness scattering is much larger than that on surface impurity one. It express the role of surface roughness scattering by low surface quality.

\section{CONCLUSION}

To summarize, in the present paper we have developed a theory for the transport of the 2DEGs at low temperature and high carrier density in Gaussian heavily-doped $\mathrm{ZnO}$ surface quantum wells. Our theory takes adequate account of the effect from large spontaneous polarization of $\mathrm{ZnO}$. It turns out that the quality of a $\mathrm{ZnO}$ accumulation layer depends strongly on its polarity. The large polarization charges bound on the $\mathrm{ZnO}$ surface lead to a remarkable decrease in all partial mobilities of electrons confined in the O-polar face ZnO SFQW, whereas an increase in the Zn-polar face one. It stress that the accumulation layer only is formed at Zn-face when the surface density has to be the high value. Our theory will be useful for the study the transport properties in the realistic $\mathrm{ZnO}$ SFQWs systems. Particularly, an adequate explanation will be published soon for the experimental results existing as a challenge for decades.

\section{REFERENCES}

[1] M. H. Huang, S. Mao, H. Feick, H. Yan, Y. Wu, H. Kind, E. Weber, R. Russo, and P. Yang, Science 292 (2001) 1897.

[2] K. Ellmer, J. Phys. D 34 (2001) 3097. 
[3] D. C. Look, Semicond. Sci. Technol. 20 (2005) S55.

[4] T. Makino, Y. Segawa, M. Kawasaki, and H. Koinuma, Semicond. Sci. Technol. 20 (2005) S78.

[5] Y. Goldstein and Y. Grinshpan, Phys. Rev. Lett. 39 (1977) 953.

[6] Y. Grinshpan, M. Nitzan, and Y. Goldstein, Phys. Rev. B 19 (1979) 1098.

[7] M. Nitzan, Y. Grinshpan, and Y. Goldstein, Phys. Rev. B 19 (1979) 4107.

[8] G. Yaron, A. Many, and Y. Goldstein, J. Appl. Phys. 58, 3508 (1985).

[9] J. Lindhart, M. Scharff, and H. E. Schiott, Kong. Danske Vid. Selsk., Mat.-Fis. Medd. 33 N. 14 (1963).

[10] V. Bogatu, A. Goldenblum, A. Many, and Y Goldstein, Phys. Status Solidi B 212 (1999) 89.

[11] G. Yaron, J. Levy, Y. Goldstein, and A. Many, J. Appl. Phys. 59 (1986) 1232.

[12] A. Dal Corso, M. Posternak, R. Resta, and A. Baldereschi, Phys. Rev. B 50 (1994) 10715.

[13] F. Bernardini, V. Fiorentini, and D. Vanderbilt, Phys. Rev. B 56 (1997) R10024.

[14] M. W. Allen, P. Miller, R. J. Reeves, and S. M. Durbin, Appl. Phys. Lett. 90 (2007) 062104.

[15] L. Tuan, N. V. Minh, and N. T. Tien, Comm. in Phys. 18 (2008) 81-87.

[16] D. N. Quang, V. N. Tuoc, N. H. Tung, N. V. Minh, and P. N. Phong, Phys. Rev. B 72 (2005) 245303.

[17] D. N. Quang, N. H. Tung, V. N. Tuoc, N. V. Minh, H. A. Huy, and D. T. Hien, Phys. Rev. B 74 (2006) 205312.

[18] T. Ando, A. B. Fowler, and F. Stern, Rev. Mod. Phys. 54 (1982) 437.

[19] N. T. Tien, L. Tuan, and D. N. Quang, Comm. in Phys. 18 (2008) 88-94.

[20] A. Gold, Phys. Rev. B 35 (1987) 723.

[21] R. M. Feenstra and M. A. Lutz, J. Appl. Phys. 78 (1995) 6091.

Received 15 June 2008. 